DOI: 10.4274/jarem.galenos.2020.3442

J Acad Res Med 2020;10(2):180-4

\title{
Topographic Evaluation of Acute Isolated Unilateral Thalamic Infarctions on Diffusion-weighted Imaging
}

\author{
(1) Sebahat Nacar Doğan \\ University of Health Sciences Turkey, Gaziosmanpaşa Training and Research Hospital, Clinic of Radiology, İstanbul, Turkey
}

Cite this article as: Dogan, Sebahat Nacar. Topographic Evaluation of Acute Isolated Unilateral Thalamic Infarctions on Diffusion-weighted Imaging. J Acad Res Med 2020;10(2):180-4

\begin{abstract}
Objective: The thalamus plays a major role in regulating arousal, consciousness, and activity. Distinct vascular distribution of the thalamus causes different syndromic presentations of thalamic nuclei infarctions. During the evaluation of acute thalamic infarction, it is important to determine the thalamic vascular zone that is affected. This study aimed to assess the topography of acute isolated unilateral thalamic infarction on diffusionweighted imaging, and to investigate the distribution of classic and variant type thalamic infarctions.

Methods: The imaging database of the 336 consecutive patients with acute thalamic infarction admitted to the radiology department between January 2015 and February 2020 were retrospectively reviewed. Specifically, patients with acute isolated unilateral thalamic infarction were included. The most affected thalamic territory, variant/classical territory rates, and the comparison of age and gender were evaluated.

Results: A total of 141 patients (classic territory group: 104, variant territory group: 37 ) were reviewed. The ratio of affected classic territory to variant territory was 2.8. Affected classic territories were inferolateral $(n=68)$, anterior $(n=25)$, paramedian $(n=11)$, and posterior $(n=0)$. Affected variant territories were posterolateral $(n=18)$, central $(n=13)$, and anteromedian $(n=6)$. Comparing the patients in both groups, age, sex, and side were similar, $p=0.435, p=0.71$, and $p=0.85$, respectively. Relevant arteries did not have stenosis in $96.2 \%$ of patients, and no significant difference was observed between both groups, $\mathrm{p}=0.631$.

Conclusion: In isolated acute unilateral thalamic ischemia, the ratio of the affected variant to the classic territory was approximately $1 / 3$. Therefore, during the radiologic evaluation of acute thalamic ischemia, a variant thalamic territory should be considered in the presence of infarction that does not fit the classic territory, in order to avoid clinical-radiological discrepancies.
\end{abstract}

Keywords: Thalamus, infarction, arteries, magnetic resonance imaging

\section{INTRODUCTION}

Thalamic infarctions constitute approximately $11 \%-14 \%$ of acute ischemic strokes of the posterior circulation, most of which are unilateral (1). Thalamic infarctions are classified traditionally into four groups based on the territories supplied by four main arteries: anterior, paramedian, inferolateral, and posterior. This classification was initially based on neuroanatomic and neuropathologic data. However, this classification is recently based on imaging techniques, especially diffusion-weighted imaging (DWI), in the acute stage (2).

The thalami are fed by perforating branches of the anterior and posterior circulation (3). The territories of the thalamus can be subdivided traditionally into four groups based on the supplying arteries: anterior, paramedian, inferolateral, and posterior (4-6). The anterior territory is supplied by the tuberothalamic arteries (polar artery), which is the largest branch of the posterior communicating artery (PComA).

ORCID IDs of the authors: S.N.D. 0000-0003-1512-5060. 
of 141 patients (77 females and 64 males with a mean age of $66.1 \pm 12.8)$ of the 336 investigated DWI. Then, the final population was divided into two groups: classic group $(n=104)$ and variant group $(n=37)$.

\section{Imaging Protocols}

DWls were obtained using two 1.5 T-magnetic resonance imaging units (GE Signa HDxt and Signa Explorer; GE, Milwaukee, WI, USA). DWIs were acquired in the axial plane with parameters field of view: $25 \mathrm{~mm}$, repetition time: $5000 \mathrm{~ms}$, echo time: $100 \mathrm{~ms}$, acquisition time: 1 , number of excitations: 1 , and $b$ values of 0 and $1000 \mathrm{~s} / \mathrm{mm}^{2}$, isotropically weighted. DWI yielded 20 contiguous slices that were $7 \mathrm{~mm}$ thick and axial-oblique. Apparent diffusion coefficient (ADC) map was automatically generated from the DWI $a t b=0$ and $b=1000 \mathrm{~s} / \mathrm{mm}^{2}$. We checked the ADC maps to ensure that "real" diffusion disturbance occurred. A visual evaluation was performed. Besides, computed tomography angiography (CTA) and magnetic resonance angiography (MRA) were evaluated to investigate the relevant arteries of the thalamus.

\section{Imaging Analysis}

All the DWls were evaluated by a radiologist with significant experience in neuroradiology (S.N.D. with 11 years in neuroradiology) on a PACS imaging workstation. The radiologist was blinded to the neurologic symptoms during the retrospective imaging review.

All the DWls were reviewed with regard to the location of the thalamic infarction based on previous templates of classic and variant thalamic territories.

In CTA or MRA examinations, the stenosis of relevant arteries was evaluated.

\section{Topography of Thalamic Infarctions}

Classic thalamic infarctions were assigned four vascular zones based on previously published territory templates (3-5): anterior, paramedian, inferolateral, and dorsal (Figure 1). Isolated posterior territory infarction was not observed in this study. Therefore, to depict the posterior territory, posterior territory infarction accompanying to PCA infarction was used was shown in Figure 1d.

Variant thalamic infarctions were assigned into three vascular zones based on previously published territory templates $(2,7)$ : anteromedian, central, and posterolateral (Figure 2).

The relevant arteries were basilar artery, PCA, and PcomA.

\section{Statistical Analysis}

IBM SPSS version 22.0 software was used for the data analysis. Normality checks were performed by the Shapiro-Wilk test, and by drawing histograms, $\mathrm{Q}-\mathrm{Q}$ plots, and box plots. Data were expressed as mean, standard deviation, minimum, maximum, frequency, and percentage. The two categories of non-normally distributed variables were analyzed using the Mann-Whitney $\mathrm{U}$ test. $\mathrm{T}$ test was used to compare the nominal variables. The significance level was taken as $p<0.05$ and bidirectional 


\section{RESULTS}

The final cohort consisted of 141 patients (41.9\%) with isolated unilateral thalamic infarctions. Thalamic infarctions were located in classic territories in 104 (73.8\%) patients and in variant territories in $37(26.2 \%)$ patients. The ratio of affected classic territory to variant territory was 2.8 .

In the total cohort, 64 (45.4\%) patients were males and 77 (54.6) were females. The thalamic infarction was located on the right side in 59 patients (41.8\%) and on the left side in 82 (58.2\%). Comparing the patients in the classic and variant groups, the age, sex, and affected side were similar for both, with $p=0.435,0.761$, and 0.852 , respectively.

The relevant artery was investigated for 80 (56.7\%) patients. CTA was performed for $84.8 \%$ of patients $(n=67)$, and MRA for $15.2 \%$ $(n=12)$. No stenosis was observed in 77 patients (96.2\%). There was more than $50 \%$ stenosis in 2 patients $(2.5 \%$ ), and $<50 \%$ stenosis in one patient (1.3\%). No significant difference in relevant artery stenosis was observed between the classic and variant groups, $\mathrm{p}=0.631$.

Table 1 summarizes the patient demographics.

Regarding the vascular territory of the thalamus, the anterior territory was involved in 25 patients (17.8\%), paramedian in
11 (7.8\%), inferolateral in 68 (48.2\%), posterior in 0 (0.0\%), anteromedian in 7 (4.9\%), central in 12 (8.5\%), and posterolateral in 19 (13.5\%) patients. While infarctions were most commonly identified in the inferolateral territory, the posterior territory was not involved in any of the patients.

Regarding the classic vascular territory of the thalamus, the anterior territory was involved in 25 patients (24\%), paramedian in 11 (10.6\%), inferolateral in 68 (65.4\%), and posterior in $0(0.0 \%)$. Inferolateral territory was the most frequently affected classic territory. However, the isolated posterior territory infarction was not observed in any patient.

Regarding the variant vascular territory of the thalamus, the anteromedian territory was involved in 6 patients (16.2\%), central in 13 (35.1\%), and posterolateral in 18 (48.7\%). The posterolateral territory was the most frequently affected variant territory.

Table 2 shows the distribution of the thalamic infarctions with vascular zones.

\section{DISCUSSION}

In the present study, the topographic pattern of isolated unilateral thalamic infarctions on DWIs was evaluated based on the relevant vascular zones. Several interesting findings were obtained. First, isolated unilateral thalamic infarctions constituted $41.6 \%$ of all
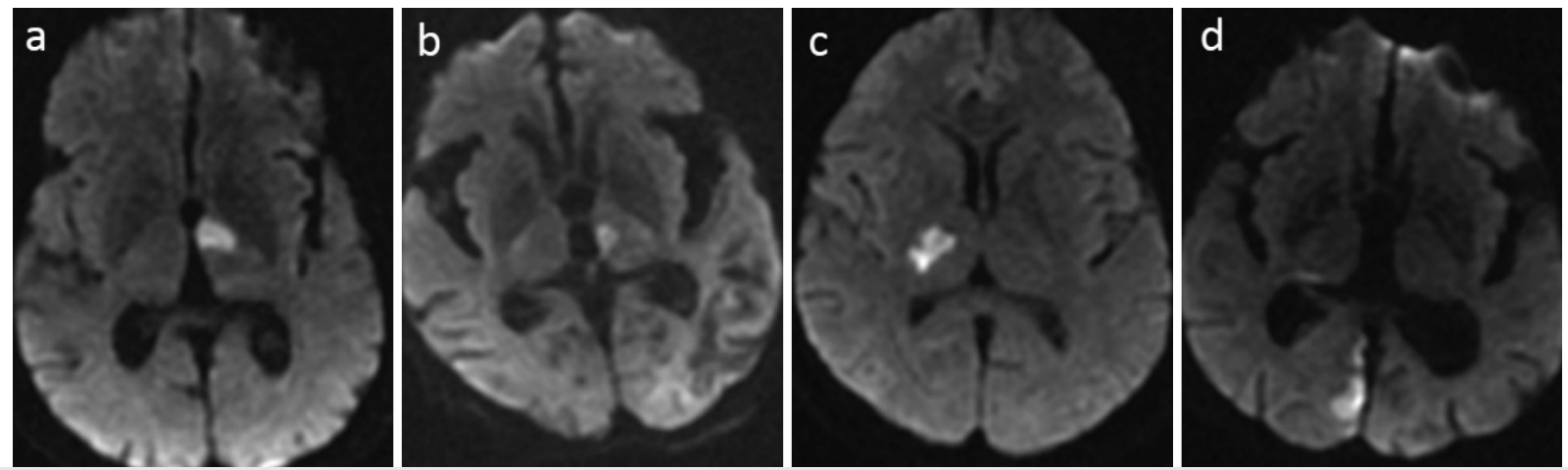

Figure 1. The classic territories of the thalamus on the DWI: anterior (a), paramedian (b), inferolateral (c), and posterior (d). The posterior territory infarction (d) was depicted from the PCA territory infarction because an isolated posterior territory infarction was not detected DWI: Diffusion weighted imaging, PCA: posterior cerebral artery
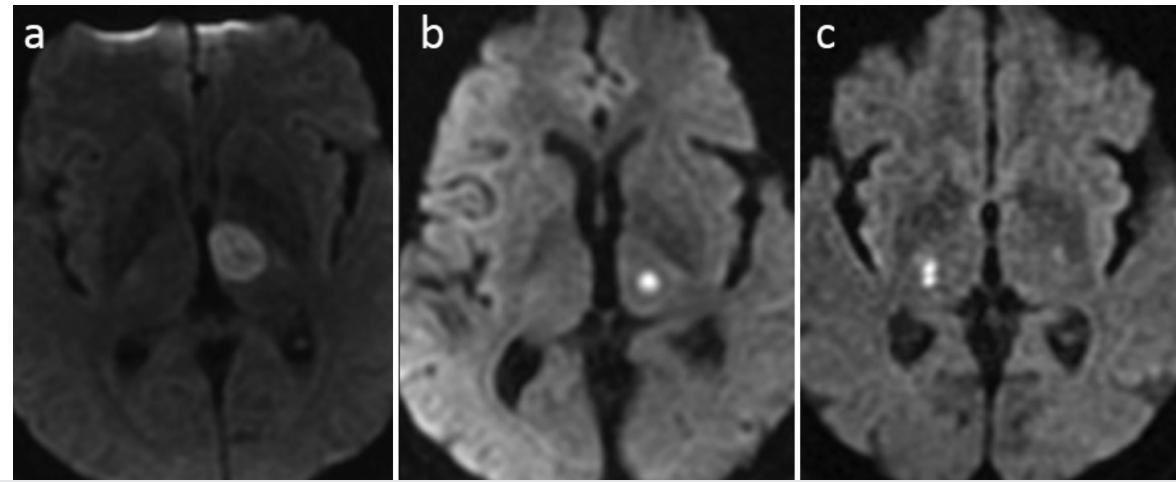

Figure 2. The variant territories of the thalamus on the DWI: anteromedian (a), central (b), and posterolateral (c) DWI: Diffusion weighted imaging 
most, consistent with previous studies (8-12). Besides, while the isolated paramedian territory was observed, the isolated posterior territory infarction was not detected. Although this result was consistent with the findings of Pezzini et al. (10), it was contrary to those of Wang et al. (8). The isolated posterior territory infarctions are the least common infarction of the thalamus. The posterior territory of the thalamus has rich anastomosis; therefore, infarction that locates only the posterior territory could be very rare. Posterior territory infarctions are usually a part of the PCA territory infarction (13-16). In addition, there may be some difficulties especially in differentiating between the classic posterior territory and variant posterolateral territory. The previous studies also did not separately describe the variant territories.

There are few reports that investigated the variant territories of the thalamus $(2,7)$. Kumral et al. (7) evaluated the acute multiple variant type thalamic infarction and they reported that the most commonly affected variant territory was posterolateral in unilateral thalamic infarction. Carrera et al. (2) investigated the isolated variant thalamic infarction. They reported the distribution of affected variant territories such as the anteromedian ( $n=9)$, posterolateral $(n=8)$, and central $(n=4)$. In present study, the most frequently affected variant territory was posterolateral, consistent with results of Kumral et al. (7). However, the least frequently affected variant territory was anteromedian, contrary to the findings of Carrera et al. (2). The frequency of the affected variant territory in the present study was different from previous studies. The discrepancy regarding the frequency of the affected variant territory among different studies may be as a result of the small sample size. Therefore, variant thalamic infarction needs to be evaluated in large series.

In addition, in this present study that evaluated relevant arteries were by CTA or MRA, large artery disease was not observed in both the sclassic and variant group. Therefore, this result can support the fact that variant territory can be as a result of variant vascular distribution rather than border-zone ischemia. Both Kumral et al. (7) and Carrera et al. (2) also reported that the most frequent cause of stroke was cardioembolism, and not large artery disease.

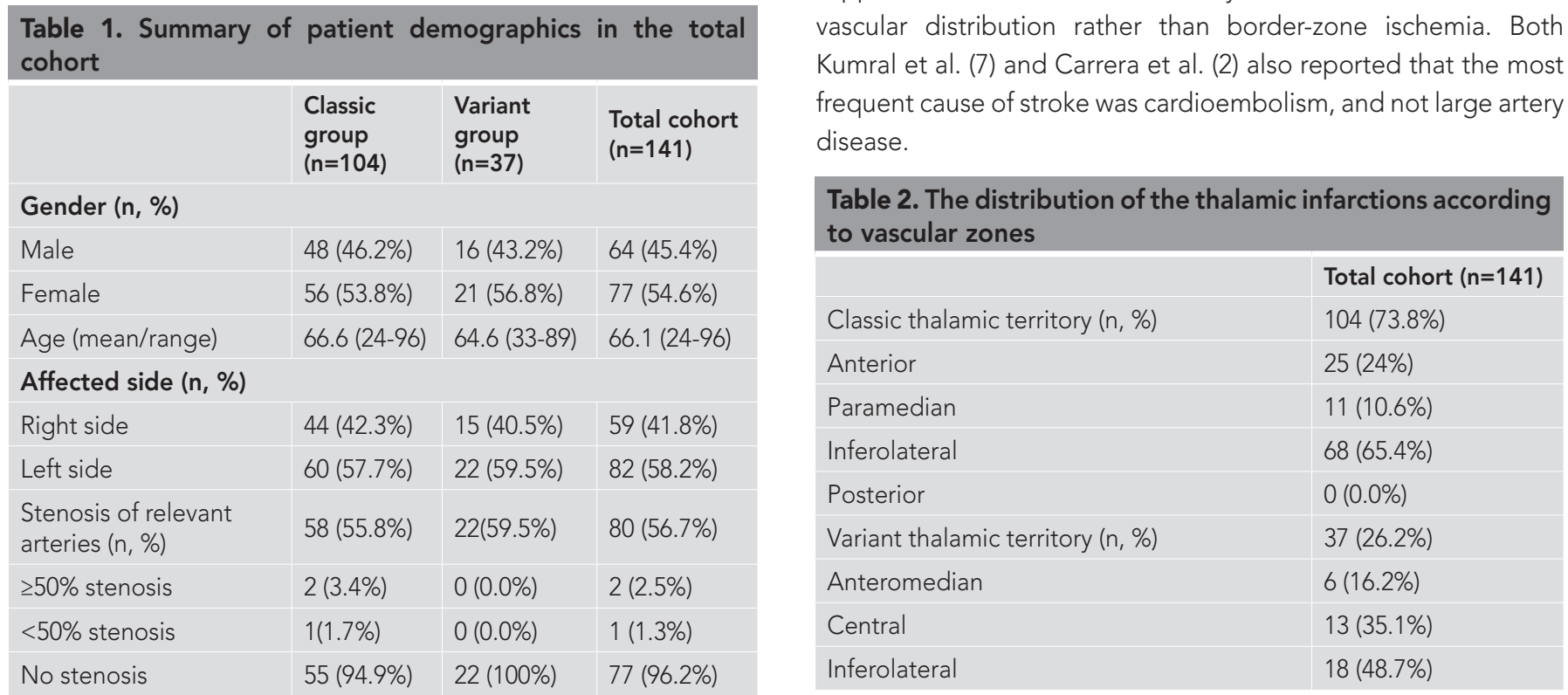




\section{Study Limitations}

Several limitations to this study need to be acknowledged. First, the study was retrospective and is subject to all the limitations of this study design. Second, it was a single-center study. Third, only a small number of infarctions were present for certain vascular territories. Forth, the CTA or MRA examination could not be performed in all the patients.

\section{CONCLUSION}

The affected territory in acute isolated thalamic infarction can be in the variant group in approximately $1 / 3$ of patients. Regarding the classic and variant groups, the most frequently affected territories were inferolateral and posterolateral, respectively. Therefore, in daily practice, during the evaluation of acute thalamic infarction on DWI, the infarction area needs to be sought for more carefully, whether it fits the classic territory or not. This is because an accurate topographic evaluation is important to avoid clinicalradiological discrepancies.

Ethics Committee Approval: Ethics committee approval was received for this study from the Ethics Committee of Taksim Training and Research Hospital (approval number: 81, date: 19/05/2020).

Informed Consent: Informed consent was waived.

Peer-review: Externally peer-reviewed.

Financial Disclosure: The authors declared that this study has received no financial support.

\section{REFERENCES}

1. Caplan L, Chung CS, Wityk R, Glass T, Tapia J, Pazdera L, et al. New England medical center posterior circulation stroke registry: I. Methods, data base, distribution of brain lesions, stroke mechanisms, and outcomes. J Clin Neurol 2005; 1: 14-30.
2. Carrera E, Michel P, Bogousslavsky J. Anteromedian, central, and posterolateral infarcts of the thalamus: Three variant types. Stroke 2004; 35: 2826-31.

3. Tatu L, Moulin T, Bogousslavsky J, Duvernoy H. Arterial territories of the human brain cerebral hemispheres. Acta Neurol Scand 1998; 50: 1699708.

4. Schmahmann JD. Vascular syndromes of the thalamus. Stroke 2003; 34: 2264-78.

5. Li S, Kumar Y, Gupta N, Abdelbaki A, Sahwney H, Kumar A, et al. Clinical and Neuroimaging Findings in Thalamic Territory Infarctions: A Review. J Neuroimaging 2018; 28: 343-9.

6. Bogousslavsky J, Regli F, Uske A. Thalarnic infarcts: Clinical syndromes, etiology, and prognosis. Neurology 1998; 38: 837-48.

7. Kumral E, Deveci EE, Çolak AY, Çağında AD, Erdoğan C. Multiple variant type thalamic infarcts: Pure and combined types. Acta Neurol Scand 2015; 131: 102-10.

8. Wang $X$, Fan YH, Lam WWM, Leung TWH, Wong KS. Clinical features, topographic patterns on DWI and etiology of thalamic infarcts. J Neurol Sci 2008; 267: 147-53.

9. Song YM. Topographic patterns of thalamic infarcts in association with stroke syndromes and aetiologies. J Neurol Neurosurg Psychiatry 2011; 82: 1083-6.

10. Pezzini A, Del Zotto E, Archetti S, Albertini A, Gasparotti R, Magoni M, et al. Thalamic infarcts in young adults: relationship between clinicaltopographic features and pathogenesis. Eur Neurol 2002; 47: 30-6.

11. Lee E, Kang DW, Kwon SU, Kim JS. Posterior cerebral artery infarction: diffusion-weighted MRI analysis of 205 patients. Cerebrovasc Dis 2009; 28: 298-305.

12. Georgiadis AL, Yamamoto Y, Kwan ES, Pessin MS, Caplan LR. Anatomy of sensory findings in patients with posterior cerebral artery territory infarction. Arch Neurol 1999; 56: 835-8.

13. Neau JP, Bogousslavsky J. The syndrome of posterior choroidal artery territory infarction. Ann Neurol 1996; 39: 779-88.

14. Bordes S, Werner C, Mathkour M, McCormack E, Iwanaga J, Loukas M, et al. Arterial supply of the thalamus: A comprehensive review. World Neurosurg 2020; 137: 310-8.

15. Saeki N, Shimazaki K, Yamaura A. Isolated infarction in the territory of lateral posterior choroidal arteries. J Neurol Neurosurg Psychiatry 1999; 67: 413-5.

16. Neau JP, Bogousslavsky J. The syndrome of posterior choroidal artery territory infarction. Ann Neurol 1996; 39: 779-88. 